\title{
Research on the Construction of E-commerce Ecosystem
}

\author{
Jianhua Zhang, Fangfang Li, Ke Liu \\ Department of Management Engineering, Zhengzhou University, Zhengzhou, China \\ 904822596@qq.com
}

Keywords: E-commerce, Ecosystem, Model Construction, System Model, Ecological Balance

Abstract: In recent years, the e-commerce industry has become the biggest boost to the growth of the national economy.How to build a good e-commerce environment is especially important for promoting the development of the e-commerce industry. The e-commerce ecosystem actively promotes the development of the e-commerce industry. This paper analyzes the current e-commerce market conditions, starting from the characteristics of the e-commerce system, and combining the actual situation with the e-commerce ecosystem. And the research and exploration of the composition, construction, and operation of the system have been carried out. Firstly, the paper analyzes the components and characteristics of the e-commerce ecosystem, and then presents the main structure and hierarchy of the e-commerce ecosystem by dividing the e-commerce ecological chain and ecosystem. Secondly, an e-commerce ecosystem model based on ecosystem theory is established, and the feasibility of the model is also expounded; finally, this paper summarize the composition and operation mode of the e-commerce ecosystem, and make relevant recommendations.

\section{Introduction}

With the development of the Internet and the gradual maturity of computer technology, e-commerce has emerged as the times require, which has greatly changed people's way of life. Since e-commerce entered China in the 1990s, it has developed rapidly. Many e-commerce companies have sprung up. In order to adapt to the changes of the market, traditional enterprises have also taken this opportunity to transform. The arrival of e-commerce has broken the traditional business model, expanded the consumer market, and drove the growth of China's economy. Especially after China's "One Belt, One Road" initiative, e-commerce has become a link connecting the world economy. In the future when the market prospect is so good, with the continuous development of its own business, e-commerce enters a new stage of intensive innovation and rapid expansion (Lili Sun, 2016). Therefore, a good e-commerce ecosystem is even more important for the e-commerce industry. The survival of the fittest is not only the survival rule of nature, but also applies to the e-commerce industry. The development of any thing is not smooth sailing. Although e-commerce has flourished in recent years, the competition has become increasingly fierce. In this process, many enterprises have been eliminated, and the surviving is to adapt to market demand, and constantly innovate and develop. These successful e-commerce 
companies can make the operation of the enterprise in a virtuous circle.

\section{Research Purposes and Significance}

E-commerce is not the first to enter the Chinese market, but the development trend is very good. In just over a decade, the business model and people's consumption patterns have been greatly changed. E-commerce is impacting all aspects of social and economic production with great vitality. It can be said that the e-commerce industry has made a significant contribution to the growth of China's economy, and today's commercial competition is increasingly manifested as a competition between the platform-based business ecosystem (Liubo Hu, Bo Yang, Sheng Cao, 2017) which is a stable business ecosystem. It is a necessary prerequisite for the development of enterprises. In recent years, the development of e-commerce in China has been rapid very much. However, many small and medium-sized e-commerce companies have been drowning in the ever-changing market torrents. The reasons for these closed enterprises are not in a benign e-commerce ecosystem, without the influence of the external environment, the establishment of a dynamic, adaptable internal environment, so as not to achieve the ability to co-evolve with other enterprises, and ultimately lead to inoperability. This paper studies the basic composition and operation mode of the e-commerce ecosystem, and strives to help e-commerce companies understand the importance of the internal and external environment and help enterprises to develop sustainable development strategies based on the business ecosystem (Huiling Xiao, Zizi Li, Wenkang Du, Yuhang Lv, 2017).

\section{Overview Of The E-Commerce Ecosystem}

The e-commerce ecosystem is not an independent concept, but a product of the intersection of disciplines. Therefore, to establish an e-commerce ecosystem, we must understand the definition and characteristics of the e-commerce ecosystem. This is also the main research content of this chapter.

\subsection{Definition of the e-commerce ecosystem}

The commercial ecosystem was first proposed by Moore. He believes that the activities of enterprises are similar to those of nature. They follow the rules of survival of the fittest, evolve constantly, and finally reach a state of balance and achieve a stable cycle of ecosystems. E-commerce is a relative concept. The ecosystems created by different standards and classifications are also different (Yihai Wang, 2017). The following definitions are adopted: E-commerce ecosystem refers to the organizations that interact with each other in the Internet and e-commerce environment. Enterprise organizations (including e-commerce companies, suppliers, consumers, logistics companies, financial institutions, third-party payment platforms, certification bodies, platform providers, advertising companies, software companies, etc.) rely on core capabilities and complementary advantages and the exchange of logistics, capital flows and information flows to achieve a complex economic group formed by value added (Hengliang Wu, Weiwei Zhang,2011). Among them, e-commerce enterprises, consumers, suppliers, financial institutions and other members have formed a value chain. The differentiated value chains constitute a value network, and information, funds and materials flow through the members of the value network.

\subsection{Characteristics of the e-commerce ecosystem}

The subject, environment and relationship are the main components of the e-commerce ecosystem. The relationship between these three parts gives following characteristics to the 
e-commerce ecosystem: openness, co-evolution, nonlinearity, autonomy, and self-adaptation (Xiumin Ye,2010). The e-commerce ecosystem is an open and self-operating ecosystem (Chonghui $\mathrm{Li}, 2017)$. The exchange of matter, information and energy between the outside world and the main body is the source of power for the continuous operation of the entire system. The interaction between subsystems of e-commerce is non-linear, and the role of subsystems is not expressed by simple additions. Its nonlinear effect originates from the internal, due to the initiative and adaptability of the subject. The process of constantly adjusting and changing the system is essentially the ideal state of achieving dynamic balance. The stability of the e-commerce ecosystem has an important impact on the healthy development of various groups in the system (Chunfa Li, Lipan Feng, Fangxu Han, Yunlong Cheng, 2015). Unbalance is the vitality of the system to adapt to complex environments, which is the independent balance of the system. In addition, within a certain scope, the adaptive function of the e-commerce ecosystem enables all individuals to purposefully conduct independent adaptive learning and evolution according to the characteristics of their own small environment.

\section{E-Commerce Ecosystem Model Construction}

E-commerce companies exhibit various competitive relationships due to resources, capital, and market and production capacity. In order to achieve long-term production operations, enterprises must seek cooperation, and on the basis of the network platform, the e-commerce ecosystem is gradually formed (Yaode Wang, Qibin Xu, 2018). Through analogy, we can know that the functional goals and structural components of the e-commerce ecosystem are similar to those of the ecosystem. We can understand the activity rules of e-commerce companies according to the survival mode and evolution process of species in nature, at the same time, analyze various influencing factors to establish a model, in order to explore an ecosystem which is suitable for the long-term development of e-commerce enterprises.

\subsection{Overview of ecosystem theory}

In ecology, an ecosystem is the same whole formed by all the organisms (ie, biomes) living together in a certain space due to continuous material circulation and energy flow (Xiangxian Zhang, Xu Zhang, Xu Zheng,2010). Like the species in the ecosystem, every species wants to survive. In addition to the internal cooperation in the population, it also has to achieve mutual benefit with other populations. It is the same for trading entities in e-commerce. They want to continue to develop and need to reach consensus with relevant enterprises and institutions, seek common ground while reserving differences and pursue a win-win situation. This relationship makes the active individual regularly form a certain structure and become a whole. This whole and its environment form a specific system, and it also known as an e-commerce ecosystem. The introduction of ecosystem theory into e-commerce system restores the essence of e-commerce industry dynamics, and at the same time reflects the evolving process of e-commerce system, visualizing abstract things, helping enterprises to establish a good industrial environment and promoting the long-term development of enterprises. The ultimate goal of the e-commerce ecosystem is also to achieve system balance and co-evolution of the internal population of the system.

\subsection{Construction principles}

Building a healthy e-commerce ecosystem is a complex project that requires consideration of multiple factors. The goal is to help e-commerce companies continue to grow in the ever-changing 
market, and the following principles should be followed in the process of building an e-commerce ecosystem.

(1) The principle of openness

Similar to the natural ecosystem, there should be many producers, consumers, decomposers and transmitters in the e-commerce ecosystem, and these main parts are not static and need to be updated as the external environment changes. An open system is needed to facilitate the entry of other e-commerce entities, improving system stability and "metabolization" functions.

(2) Systematic principles

The e-commerce ecosystem arranges the various parts in an orderly manner to form a hierarchical, dynamic and complex whole. Each component of the ecosystem has its own function, and follows the systemic principle, making each subsystem According to their own functions, they are in different niches to ensure that the entire e-commerce ecosystem can operate stably.

(3) The principle of interaction

The factors in the e-commerce ecosystem interact with each other. If the divisions are broken, the information flow will be interrupted, and the transmission of information in the entire ecosystem will not be realized, which will lead to the rigidity of the ecosystem. Therefore, the e-commerce ecosystem is in a process of continuous movement, mutual influence and mutual evolution, which lays a solid foundation for the continuous improvement, continuous improvement and good operation of the e-commerce ecosystem.

\subsection{Model construction}

The e-commerce ecosystem is mainly composed of e-commerce ecological subjects and internal and external ecological environments. According to the ecosystem theory, e-commerce main factors include producers, consumers, transmitters and decomposers. Within the system, e-commerce main factors can be attributed to the flow of information, logistics and capital flow throughout the system according to the nature of the commercial system. The whole system is affected by the external environment, including economic, legal, social, cultural, etc. According to the analysis of the e-commerce industry and combined with the characteristics of ecology, establish an e-commerce ecosystem model as shown in Figure 1.

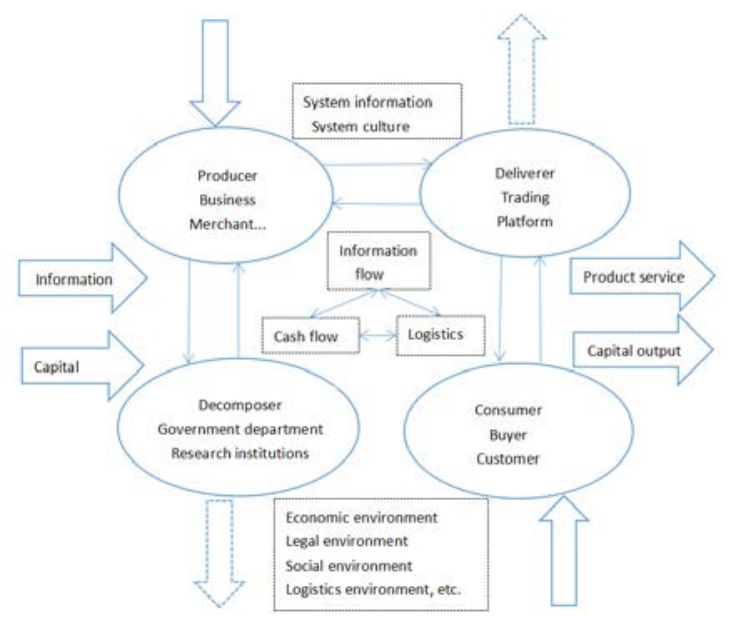

Figure 1 E-commerce ecosystem model.

In the unified model, information resources generated by market demand enter the established system model, which is the main source of material transmitted between the internal information flow, capital flow, and logistics among producers, transmitters, consumers, and decomposers. The 
producer processes these resources into products, and delivers them to the consumers through the deliverers. The success of the consumers is an important way to generate transaction funds, and the decomposers will pass the information analyzed in the process to the production. At the same time, producers and consumers feed back to the decomposers according to their own situation. In this whole process, information, capital and logistics continue to circulate, which is an important guarantee for the continuous operation and change of the e-commerce ecosystem. When various capital information enters the system, the e-commerce ecosystem will transmit to the outside of the system due to the corresponding information and culture generated by the operation, and will affect the external environment of the system. At the same time, the external environment will interact with the system due to the original influencing factors. The resulting new impact factors form a new external environment while counteracting the e-commerce ecosystem. In addition, the output of product services and capital generated in this process system is transformed into the accumulation of the external environment, laying the foundation for the evolution of the ecosystem, and making the whole system in a process of continuous movement and upgrading. Only when the subsystems maintain a coordinated and stable operating state can the e-commerce ecosystem be dynamically balanced.

\subsection{Feasibility analysis of the model}

The e-commerce ecosystem model constructed in Figure 1 is based on the laws of natural ecosystems, and has the characteristics of overall structural optimization and ecological factor coordination to ensure the feasibility of the model. First of all, the internal elements of the system model are closely related. The interaction and mutual influence of the subject factors and environmental factors form the overall structure of the model, and the purpose of optimizing the e-commerce ecosystem as a whole is realized from the perspective of macro-micro and space-time. Secondly, the e-commerce ecological factors in the system model are coordinated with each other. At the same time, the main types of e-commerce ecosystems are rich, and the information producers in the model are in the leading position of e-commerce ecological subjects, while consumers, transmitters and decomposers according to the producer's instructions and products, the related work is in a non-dominant position. Such a model structure is in line with the actual situation in reality, and it is feasible to have scientific theory to guide it.

\section{Conclusions}

The e-commerce ecosystem is a complex, open and dynamic system with internal components and interactions. Based on the previous research results, this paper analyzes the virtual ecological factors and studies the structural composition of the e-commerce ecosystem. From the perspective of ecosystem theory, it establishes the e-commerce ecosystem model and analyzes the feasibility of the model. The conclusions are as follows: (1) The healthy e-commerce ecosystem is the key to the development of e-commerce enterprises; (2) The benign development of e-commerce ecosystem needs the balance of internal and external environments to maintain; (3) Technology is the fundamental driving force for the development of e-commerce. The model established in this paper provides a theoretical reference for the Se-commerce industry, and it is hoped to help e-commerce enterprises to establish an e-commerce ecosystem. Unfortunately, the article does not specifically quantify the model, and there is no specific data to support it. This can be further studied and discussed as a future research direction. 


\section{References}

[1] Chonghui Li, J., 2017. Construction of e-commerce platform enterprise ecosystem, Industry and Technology Forum. 16(12), pp.278-279

[2] Chunfa Li, Lipan Feng, Fangxu Han, Yunlong Cheng, J., 2015. Dynamic Evolution Game Analysis of E-commerce Ecosystem, Journal of Systems Science. 23(04), pp.75-78

[3] Hengliang Wu, Weiwei Zhang, C., 2011. Constructing an Ecosystem Study Suitable for the Healthy Development of E-commerce in China, Proceedings of Conference on Web Based Business Management (WBM2011).

[4] Huiling Xiao, Zizi Li, Wenkang Du, Yuhang Lv, J., 2017. Research on E-commerce Platform Enterprise Ecosystem and Its Strategic Evolution—Based on Enterprise Case Study, Market Modernization. (07), pp.50-52

[5] Lili Sun, J., 2016. Construction of Knowledge Ecosystem Model for E-commerce Enterprises Facing Knowledge Innovation, Information Science. 34(12),pp.141-144

[6] Liubo Hu, Bo Yang, Sheng Cao, J., 2017. Evolution Stage and Investment Strategy of Agricultural Product E-commerce Ecosystem Structure, Business Economics Research. (05),pp.67-69

[7] Xiangxian Zhang, Xu Zhang, Xu Zheng, J., 2010. Research on the Construction of E-commerce Information Ecosystem, Library and Information Work. 54(10), pp.20-24

[8] Xiumin Ye, M., 2010. E-commerce Ecosystem Research,Social Science Literature Publishing House. Beijing.

[9] Yaode Wang, Qibin Xu, J., 2018. The Construction of E-commerce Value Ecosystem, Technical Economics and Management Research. (02), pp.64-69

[10] Yihai Wang, J., 2017.E-commerce Information Service Ecosystem Application, E-commerce. (10), pp.10-11 